\title{
COMMENTARY
}

\section{Evolutionary Psychology: A View From Evolutionary Biology}

\author{
Elisabeth A. Lloyd \\ History and Philosophy of Science Department \\ Biology Department \\ Indiana University, Bloomington \\ Marcus W. Feldman \\ Department of Biological Sciences \\ Stanford University
}

Given the recent explosion of interest in applications of evolutionary biology to understanding human psychology, we think it timely to assure better understanding of modern evolutionary theory among the psychologists who might be using it. We find it necessary to do so because of the very reduced version of evolutionary theorizing that has been incorporated into much of evolutionary psychology so far. Our aim here is to clarify why the use of a reduced version of evolutionary genetics will lead to faulty science and to indicate where other resources of evolutionary biology can be found that might elevate the standard of the evolutionary component of evolutionary psychology.

\section{The Reduced View of Evolutionary Genetics}

In characterizing and defending evolutionary psychology, Ketelaar and Ellis (2000a) described the metatheoretical science on which all evolutionary psychology is built as "the general principles of genetical evolutionary theory, as outlined by W. D. Hamilton (1964) and instantiated in more contemporary 'selfish gene' theories of genetical evolution via natural and sexual selection. (See Cronin, 1991; Dawkins, 1976, 1982, 1986; Dennett, 1995; Mayr, 1983; Tooby \& Cosmides, 1992; Williams, 1966, 1992, for good overviews of the basic assumptions of modern evolutionary theory.)" (p. 4).

Their point was that evolutionary psychologists take the modern theory of evolution to provide a set of core assumptions that enable them to distinguish between plausible and implausible a priori psychological hypotheses. Ketelaar and Ellis (2000b) also later characterized the foundational metatheory of evolution as "inclusive fitness theory" (p. 62). They are fairly typical among proponents and defenders of evolutionary psychology in their views of what constitutes the foundations of modern evolutionary theory. As Foley (1995-1996) put it, evolutionary psychology claims that "human behavior and its cognitive base should be explicable in terms of enhanced inclusive fitness" (p. 195).

The immediate problem with such a representation of evolutionary biology is that inclusive fitness theory comprises a small subset of models used for special purposes in evolutionary understanding, as we shall explain later. There are many other components of evolutionary analysis that address both animal and human behavior, and although they are conceptually more intricate than inclusive fitness theory, they may be more appropriate for the exploration of human psychology.

Tooby and Cosmides (1998) seemed to advocate a broader view when they recommended "incorporating knowledge from evolutionary biology and its related disciplines-behavioral ecology, paleoanthropology, hunter-gatherer studies, and primatology-into [cognitive scientists'] repertoire of theoretical tools" (p. 195). This breadth is more apparent than real, however, as one can tell from their presentation of what they called the "primary literature" of evolutionary biology itself, in which they cited Clutton-Brock and Harvey (1979), Daly and Wilson (1983), Dawkins (1982, 1986), Krebs and Davies (1987), Williams (1966), and Williams and Nesse (1991; Cosmides \& Tooby, 1994, p. 43). Readers unfamiliar with evolutionary theory will not recognize that this list, with the exception only of Williams, encompasses a particular small corner of applications of evolutionary thought and does not in fact represent the foundational aspects of evolutionary genetics and theory. Nowhere do we see citations to fundamental re- 
search in evolutionary genetics or evolutionary biology such as Fisher (1918, 1930), Wright (1931), Kimura (1969), Dobzhansky (1970), or Lewontin (1974) or to important textbooks such as Hartl and Clark (1997), Futuyma (1998), and Falconer and Mackay (1996). So in the case of both Ketelaar and Ellis (2000a) and Tooby and Cosmides, nowhere are the real foundations of evolutionary thought cited or appealed to.

\section{Harm Done by the Reduced View of Evolutionary Genetics}

We claim that serious mischief is done to the psychological sciences through acceptance of this reduced, inaccurate presentation of the modern theoretical basis of evolutionary analysis.

One result of the presentation of evolutionary theory as equivalent to inclusive fitness theory is that it focuses all attention on adaptation as a result of optimization of inclusive fitness, which in many cases is not the best way to represent the evolutionary dynamic (Cavalli-Sforza \& Feldman, 1978). This is especially true, for example, in cases of sexual or fertility selection, where fitness is properly assigned to a mating pair rather than individual genotypes. When these forces are stronger than the forces of differential survival ability, predictions made by maximizing inclusive fitness will not yield correct results. Indeed, under these modes of selection, the mean fitness of the population is not maximized by the process of evolution at all (see Pollack, 1978, and Reduced Adaptationism).

Some evolutionary psychologists have recognized that the picture of evolution that results from viewing evolutionary theory as equivalent to inclusive fitness theory is impoverished and likely to be empirically inadequate. For instance, in commenting on Ketelaar and Ellis's (2000a) defense of evolutionary psychology, evolutionary psychologist Miller (2000a, 2000b) argued for the importance of including sexual selection models, whereas Caporael and Brewer (2000) promoted the necessity of incorporating various multilevel selection models into the corpus of evolutionary genetics from which evolutionary psychology draws. These are important extensions toward a more adequate view of evolutionary genetics, on which we now elaborate.

Contrary to the claim of Ketelaar and Ellis (2000a), for example, multilevel selection theory is not a version or derivation of inclusive fitness theory; rather, it is the other way around. Although inclusive fitness theory serves as the heart of most evolutionary psychologists' notion of genetics, it holds, in fact, a rather fringe position in evolutionary genetics itself. It is even unclear whether the notion of inclusive fitness is necessary to evolutionary genetics. The reason is as follows. The theory of kin selection can be made a part of the corpus of population genetics theory by regarding indi- vidual genotypic fitnesses as having an appropriate frequency-dependent structure that fits fully within Darwinian population genetic modeling. Therefore, the concept of inclusive fitness is unnecessary to explain kin selection.

Indeed, inclusive fitness models may produce a picture of the evolutionary dynamics of kin selection that is incorrect. For example, there are cases in which an analytically exact population genetic analysis accurately predicts situations where altruists and selfish individuals coexist stably, whereas under exactly the same conditions, inclusive fitness reasoning wrongly predicts that only altruists should survive (Cavalli-Sforza \& Feldman 1978).

So why is the notion of inclusive fitness used at all? If you are an evolutionist trying to describe genetic relations between individuals that are more distantly related than first-degree relatives (i.e., immediate family), the process of genotypic selection makes the computation mathematically intractable. The solution is to assume a very particular form of extremely weak selection, under which genotype (pairs of genes in an individual) frequencies can be expressed in terms of allele (single-gene) frequencies according to the assumptions of Hardy-Weinberg equilibrium. The resulting reduction in dimensionality not only allows calculations to be made in terms of probabilities of grandparent and cousin similarities, but it produces an average fitness in the population that is a function of the allele frequencies alone rather than of the full genotype frequencies (Hamilton, 1964). This is the attraction and utility of the inclusive fitness type of genic modeling. Note that it is a special-use model and cannot be used to represent other types of evolutionary genetic systems. Its focus on allele frequencies has led to its identification with selfish gene theory, and it is important that psychologists understand the limited range of inclusive fitness model use in evolutionary modeling. In his overview introduction to his 1964 article published in his 1996 book, Hamilton wrote:

My long endeavour to generalize a maximizing property of the classical selection model was vitiated by my relatedness coefficient being only properly defined if there were no selection, which was obviously not true in my model. Indeed, selection was the whole point. So the "proof" I came up with was really only suggestive to what would happen, not a watertight demonstration. Nevertheless, it was easy to see that the argument must apply with increasing accuracy as selection in the model was made weak. (p. 27)

Indeed, at the beginning of his original article, Hamilton (1964) wrote:

At least as we humans perceive the matter, it is not genes but we-whole diploid organisms - that make 
the decisions, so I had been delighted to find something approaching an individualistic view that I could justify for whole genotypes. (p. 2)

Not only is it incorrect to claim that inclusive fitness is the foundation of modern evolutionary theory, it is incorrect even within the restricted framework of behavioral evolution, where it cannot handle sexual selection, selection based on two or more interacting genes, or genotype-environment interactions, such as those mediated by cultural evolution. Thus, few practicing evolutionary geneticists would take inclusive fitness to be the core of their discipline.

\section{An Expanded View of Behavioral Evolution}

There are several aspects of evolutionary science that make crucial differences to evolutionary outcome and dynamics and that have been virtually ignored by most evolutionary psychologists. We now review several of these, contrasting them with the usual assumptions presented by evolutionary psychologists.

As noted by Ketelaar and Ellis (2000a), evolutionary psychology has involved several assumptions about the evolutionary process as it has affected human beings. The traits explained by evolutionary psychology, for example, are by definition universal; they are traits shared by every human being. Also, Tooby and Cosmides (1992), as well as Symons (1995) and Buss (1995), committed themselves to the view that human psychology has evolved to be highly modular. This is a direct consequence of their approach, which emphasizes the existence of environmental problems to be solved in the human evolutionary past: Problems and challenges faced our ancestors, and individual "modules" controlling behavior evolved through the selection pressure manifest in these modules. All of this is part of the general approach in evolutionary psychology, which is one of adaptationism. Adaptationism involves the assumption that any trait of interest is biological in origin and that it must have evolved to solve a particular environmental problem. Although each of these assumptions has been key to most evolutionary psychology as it has been practiced so far, we suggest here some alternative assumptions that take advantage of the full armamentarium of modern evolutionary theory.

Take the assumption of universality (i.e., the view of a species-wide evolved human nature). As Miller (2000a) pointed out, this assumption is not shared by other evolutionary biologists or by animal behaviorists. In fact, there is a weight of evidence against such an assumption, in the form of high within-population variability for most measured human behavioral traits, including the cognitive traits at stake in evolutionary psychology (Miller, 2000a, p. 43). Although this variability is often interpreted as being primarily due to genetic differences between individuals, it is also attributable to culturally transmitted differences in both the trait and the selective environment of the trait. In other words, Miller (2000b) emphasized that although selection for survival may tend to lead to universally shared, functionally specialized units, the opposite makes sense for the many adaptations that are shaped by sexual selection (e.g., peacocks' tails), among which there are large individual differences. An important possible confusion exists because of the technical genetic term heritability and its connection to the everyday notion of inheritance. Intuitively, one would think that a species-wide, shared trait would be highly heritable. However, technically, such traits have zero heritability, even though they are completely inherited. The traits considered by Cosmides and Tooby (1992), for example, would under their assumptions have zero heritability, whereas those traits that varied among individuals would have high heritability because what causes the heritability is variation among individuals.

Consider also the assumption of modularity. This amounts to the claim that cognition is more likely to consist of "many mental rules that are specialized for reasoning about various evolutionarily important domains, such as cooperation, aggressive threat, parenting, disease avoidance, predator avoidance, object permanence, and object movement" (Cosmides \& Tooby, 1992, p. 179). Evolutionary psychologists assume that human beings have a module (or set of genes) for each task that is posed by the environment:

\begin{abstract}
A central assumption of evolutionary psychology is that the human brain-mind is comprised of a large number of specialized cognitive adaptations that were shaped by natural selection over vast periods of time to solve the recurrent information-processing problems faced by our ancestors (Symons, 1995). (Ketelaar \& Ellis, 2000b, p. 61).
\end{abstract}

The relevant environment is assumed to have occurred in the late Pleistocene, and evolutionary psychologists call it the Environment of Evolutionary Adaptedness (EEA). This concept has come under strong attack. Foley (1995-1996) stated:

To argue that traits associated with the evolution of modern hunter-gatherer behavior are under genetic control, and therefore are a deeply and genetically embedded part of the EEA, leads to the conclusion that the major behavioral differences in human populations today are the result of genetic differences. This is counter to both the behavioral findings of anthropology over the last century and to the nature of intra- and interpopulation variability as it is currently understood. (p. 202) 
Shapiro and Epstein (1998) also recently challenged Cosmides and Tooby's (1994) views on modularity. They argued that Cosmides and Tooby set up a straw man in the form of the supposed commitment of cognitive psychology to domain-general problem-solving brains. Rather, Shapiro and Epstein argued there is no consensus at all with regard to the views of cognitive psychology about modularity and domain specificity. Yet Tooby and Cosmides (1994) claimed that a unique advantage of the evolutionary viewpoint is that modularity is simply derivable from evolution. Shapiro and Epstein pointed out that Cosmides and Tooby's assumption was that "organisms face a variety of adaptive problems, i.e. long-standing problems the solutions to which have required that species evolve in characteristic ways. Cosmides and Tooby conceive of adaptive problems as defining domains for which cognitive processes have evolved" (Shapiro \& Epstein, 1998, p. 174). However, Shapiro and Epstein argued that Cosmides and Tooby failed to establish that selection will favor distinct cognitive processes for the solution of every adaptive problem. Briefly, Cosmides and Tooby's argument for the inevitability of domain specificity from an evolutionary view "makes the mistake of identifying cognitive processes with the tasks or goals in which cognitive processes serve" (Shapiro \& Epstein, 1998, p. 175). As Shapiro and Epstein put the rather obvious point, "It simply does not follow from the fact that different adaptive problems require different kinds of solutions that an organism will have as many pieces of cognitive equipment as there are adaptive problems it must solve" (p. 175). Too much specificity of function may not make evolutionary sense; it may be more economical to overlap cognitive processes.

More important, a much more sophisticated view of the role of the environment is available from evolutionary theory. Niche-construction theory eliminates the notion that there is a task, independent and prior to the individual organism; on this view, it is incorrect to see evolution as fundamentally solving problems, as if environmental problems preexist the organisms, which are then subject to them as a selection pressure.

In fact, hominids have been modifying their environments for at least 3 million years, and a more correct view of human evolution would entail simultaneous evolution of the human and its environment, the latter consisting of artifacts and concepts that can be learned and improved. The organism is viewed then as part of the environment and changes in each occur during the trajectory of evolution. The changes wrought by an organism in one generation may change the environment to be faced by the next generation; this is an ecological inheritance (Laland, Odling-Smee, \& Feldman, 2000). With the advent of more sophisticated tools and techniques, the role of cultural transmission becomes central. Ex- amples of this offered by Durham (1991) are the coevolution of the gene for lactose tolerance and the cultural trait of dairying (p. 226ff) and the gene for sickle cell disease and the culturally transmitted agricultural practice of deforestation that leads to an increased incidence of malaria among the Kwa-speaking peoples of the southern forest belt of west Africa (pp. 131-145). Thus incorporation of niche construction into hominid evolutionary theory requires the recognition of cultural transmission and its consequences (Laland et al., 2000; Rozin, 2000). What is needed in this context is a more precise definition of what culture might be. It would be more appropriate to move away from the connection between genes and culture being described by statistics such as heritability and to move into a more anthropological mode, asking how the interaction between culture and artifact has driven the last 100,000 years of human evolution.

This brings us to the body of evolutionary theory that has been left out completely by the evolutionary psychologists, namely, gene-culture coevolution theory. This is a body of theory that incorporates an articulated, multidirectional coevolution between genes and culture (Boyd \& Richerson, 1985; Cavalli-Sforza \& Feldman, 1981; Durham, 1991; Feldman \& Cavalli-Sforza, 1976). Gene-culture coevolution theory does assume, in the process, that there must have been early predispositions (possibly not limited to our own hominid lineage) that had to be in place for culture to emerge. It also assumes the existence of special, hominid biological changes that permitted language to become much more sophisticated in human beings.

\section{Reduced Adaptationism}

Finally, let us turn to the assumption of adaptationism. The usual evolutionary psychology view of adaptationism is very simple and correct as far as it goes: The only way to get complex biological adaptations is through the action of natural selection on variable traits. In this simple formulation, real evolutionary constraints, such as limitations on development and genetic variety, are not mentioned. Such constraints restrict the range of subsequent adaptations, which are always contingent on prior evolutionary events (Gould, 1977). Once a complex biological adaptation exists, the only legitimate explanation of it is that it evolved by natural selection. This simple truth is then employed in a myriad of ways that are potentially highly unscientific or unverified. Note, for example, that there are two fundamental assumptions made when evolutionary psychologists appeal to this simple truth. First, they identify a human behavioral disposition and create an account of how it can be thought of as biologically caused. Second, they create an account of how that biological cause would have evolved. One question is what the data are that they offer 
to support these two concrete accounts. Lloyd (1999) noted that in support of Cosmides' (1989) famous theory about the evolution of a module for cheat detection on social contracts, the only evidence proffered was the results of a pen-and-paper test, the Wason Selection Logic Test. No efforts were made to offer evidence for the biological basis of the trait in question or for the evolutionary origins of the trait. This is simply not good enough to be regarded as part of the science of evolutionary biology.

Another problem with the reduced adaptationism usually used in evolutionary psychology is its assumption of optimization. When Richardson (2000, p. 63) criticized Ketelaar and Ellis (2000a) on these grounds, they replied that evolutionary psychology does not assume optimality. However, this is wrong. Optimization is built into inclusive fitness theory itself; individuals are assumed to maximize their own inclusive fitness, and the mean inclusive fitness of the population is also assumed to be maximized. Only those traits that increase the inclusive fitness of an organism are conceived as evolvable. Moreover, the architects of evolutionary psychology, such as Tooby and DeVore (1987), explicitly have adopted an optimality assumption in the form of their strategic modeling.

The problem with assuming optimization harks back to our remarks on genotypic versus genic selection. Under the complex dynamics of one or more genes under the assumptions of kin altruism, for example, the average fitness of the population is not necessarily increasing over time nor does the population approach a unique stable state from any starting condition (Uyenoyama \& Feldman, 1981). That is, history is important.

In most optimality arguments in evolutionary biology, the function that is optimized is an artificial construct designed by the investigator in light of his or her view of what might have been important to the organism during its evolution. This function may not bear a direct or natural relation to the underlying dynamics of genes that produce the trait. There are virtually no cases in population genetic theory, for example, where, for a trait determined by two linked interacting genes, such functions can be proven to converge monotonically to an optimum. Indeed (Lewontin, 1974), there may be many equilibria, none of which is a maximum of anything natural about the organism. Thus, the usual evolutionary psychological assumption of optimization in response to a given problem is highly problematic, from the point of view of evolutionary biology tout court.

We give one final note on the reduced adaptationism and the reduced evolutionary genetics used so often in evolutionary psychology. In a bizarre twist in the plot, evolutionary psychologists have begun to explain resistance to their theorizing as resulting from evolutionarily selected aspects of cognition. For in- stance, Young and Persell (2000) argued that critiques of evolutionary psychology often employ simplifying and erroneous assumptions about "either the mechanics of how evolution operates or the inevitable implications of evolution for understanding human behavior" (p. 218; cf. Hass et al., 2000). What we argue here is that the erroneous assumptions about the mechanics of how evolution operates lie firmly in the heads of the evolutionary psychologists themselves and not merely in their critics.

\section{Expanded Adaptationism}

We are endorsing an expanded adaptationism that uses the full resources of population genetics and behavioral biology. This would include not only frequency-dependent selection models, sexual and fertility selection models, and multilevel selection models but also includes niche construction as opposed to the fixed EEA. Niche-construction theory (Laland, Odling-Smee, \& Feldman, 1996; Laland et al., 2000; Lewontin, 1983, 1996) incorporates a dynamic of evolutionary environments, which is much more empirically and theoretically adequate to the evolutionary question; on this approach, insofar as the evolutionary process is concerned, the dynamic of evolutionary environments proceeds in concert with the changes in the organisms over time.

In an expanded adaptationism, the full range of selective forces would be taken as tools of potential evolutionary change. Thus, selection would not be thought of as viability selection alone, as it so frequently is within evolutionary psychology. Indeed, formal modeling of kin selection that leads to the inclusive fitness approach uses a fitness currency that is strictly viability. Fertility, for example, which is actually a property of a mating pair of genotypes, is much more difficult to place under this rubric.

Laboratory data indicate that only about $15 \%$ of measured lifetime fitness differences are due to viability (e.g., Lewontin, 1974); the vast majority of fitness difference comes from fertility and sexual selection. This is a problem for the evolutionary psychologist because getting the necessary measures of heritability related to sexual selection turns out be very difficult, and these measures are difficult to put back into the calculations of fitness differentials. Thus, the incorporation of mate choice, levels of polygyny, and sexual selection all change the view of the effect of natural selection acting on variability. Moreover, incorporating these important contributions to evolutionary dynamics makes it extremely difficult to represent these dynamics, even approximately in terms of inclusive fitness. In other words, the evolutionary genetics used by most evolutionary psychologists are completely in- 
adequate to the task of incorporating these factors already known to be of major evolutionary importance.

Another crucial part of an expanded adaptationism includes the possibility, indeed the likely event, that many human adaptations are, in fact, not biological adaptations at all. We only have to look at the vast collection of tools and other artifacts that archaeologists have discovered as representing the culture of our ancestors over the past few hundred thousand years. Again, the approach of the evolutionary psychologists so far has been to isolate a human behavior, assume that it is biological, and then develop a biological evolutionary account of it, preferably one in which the selective events occurred during the Pleistocene. An expanded adaptationism would challenge such an approach on several fronts. First would come the questioning of the source of the trait; it would not be assumed at the outset that it is biological in origin in any interesting way-it may be cultural, or caused by the interaction of genetic and cultural factors. Second, once a trait is established as being caused by genes, for example, circadian rhythms in the endocrine, immunological, and some neurological systems of the body, then questions can be posed about the evolutionary origins of this trait. However, it must be clear what the limitations of this kind of science are. We are in a position to demonstrate individual genetic differences between contemporary human beings, for example, and all data point to most of these differences as existing within populations that are relatively restricted in range (e.g., there are few continental-level differences, as evaluated over hundreds of genes; Barbujani, Magagni, Minch, \& Cavalli-Sforza, 1997). On the other hand, it has been extremely difficult to demonstrate that observed differences between human groups have adaptational or selectional components. Once the biological prerequisites for culture are in place, subsequent differentiation between individuals of populations may involve factors that vary at the level of genetic determination from $100 \%$ to zero. Thus, the evidentiary burden on the evolutionary psychologists for the types of claim they want to make is really quite severe and is recognized as such across the field of evolutionary genetics. Very few cases of human variation meet the standards set by our understanding of the sickle cell polymorphism in terms of genotypic fitness responses in relation to the malarial parasite.

In conclusion, the evolutionary psychology program has two serious difficulties: first, to explain the variation that exists among individuals (An Expanded View of Behavioral Evolution) and, second, to provide evidence that traits themselves exist by virtue of a selective evolutionary past (Expanded Adaptationism).

In their response to critics, Ketelaar and Ellis (2000b) claimed, following Buss, Haselton, Shackelford, Bleske, and Wakefield (1998), that there are 30 new "empirical discoveries about human psy- chology that are clearly tied to theoretical growth in evolutionary psychology" (p. 60). To demonstrate that these are (a) universal, (b) modular, and (c) adaptations would take the level of demographic analysis that led to our understanding of the sickle cell trait and malaria. It is not sufficient merely to state that a behavior is the result of psychology to have it become an adaptation. In fact, there may well be 30 traits that evolutionary psychology has usurped as being in their domain; this in itself does not explain their evolution.

\section{Note}

Elisabeth A. Lloyd, History and Philosophy of Science Department, 130 Goodbody Hall, Indiana University, Bloomington, Bloomington, IN 47405. E-mail: ealloyd@Indiana.edu

\section{References}

Barbujani, G., Magagni, A., Minch, E., \& Cavalli-Sforza, L .L. (1997). An appointment of human DNA diversity. Proceedings of the National Academy of Sciences USA, 94, 4516-4519.

Boyd, R., \& Richerson, R. J. (1985). Culture and the evolutionary process. Chicago: University of Chicago Press.

Buss, D. M. (1995). Evolutionary psychology: A new paradigm for psychological science. Psychological Inquiry, 6, 1-30.

Buss, D. M., Haselton, M. G., Shackelford, T. K., Bleske, A. L. O., \& Wakefield, J. C. (1998). Adaptations, exaptations, and spandrels. American Psychologist, 53, 533-548.

Caporael, L. R., \& Brewer, M. B. (2000). Metatheories, evolution, and psychology: Once more with feeling. Psychological Inquiry, 11, 23-26.

Cavalli-Sforza, L. L., \& Feldman, M. W. (1978). Darwinian selection and "altruism." Theoretical Population Genetics, 14, 268-280.

Cavalli-Sforza, L. L., \& Feldman, M. W. (1981). Cultural transmission and evolution. Princeton, NJ: Princeton University Press.

Clutton-Brock, T. H., \& Harvey, P. (1979). Comparison and adaptation. Proceedings of the Royal Society, London B, 205, 547-565.

Cosmides, L. (1989). The logic of social exchange: Has natural selection shaped how humans reason? Studies with the Wason Selection Task. Cognition, 31, 187-276.

Cosmides, L., \& Tooby, J. (1992). Cognitive adaptations for social exchange. In J. H. Barkow, L. Cosmides, \& J. Tooby (Eds.), The adapted mind: Evolutionary psychology and the generation of culture (pp. 163-228). New York: Oxford University Press.

Cosmides, L., \& Tooby, J. (1994). Beyond intuition and instinct blindness: Toward an evolutionarily rigorous cognitive science. Cognition, 50, 41-77.

Cronin, H. (1991). The ant and the peacock: Altruism and sexual selection from Darwin to today. New York: Cambridge University Press.

Daly, M., \& Wilson, M. (1983). Sex, evolution and behavior. Boston: Wadsworth.

Dawkins, R. (1976). The selfish gene. New York: Oxford University Press.

Dawkins, R. (1982). The extended phenotype. San Francisco: Freeman.

Dawkins, R. (1986). The blind watchmaker. New York: Norton.

Dennett, D. (1995). Darwin's dangerous idea: Evolution and the meanings of life. New York: Simon \& Schuster.

Dobzhansky, T. (1970). Genetics of the evolutionary process. New York: Columbia University Press. 


\section{LLOYD \& FELDMAN}

Durham, W. H. (1991). Coevolution: Genes, culture and human diversity. Palo Alto, CA: Stanford University Press.

Falconer, D. S., \& Mackay, T. F. C. (1996). Introduction to quantitative genetics. Harlow, England: Longman.

Feldman, M. W., \& Cavalli-Sforza, L. L. (1976). Cultural and biological evolutionary processes, selection for a trait under complex transmission. Theoretical Population Biology, 9, 238-259.

Fisher, R. A. (1918). The causes of human variability. Eugenics Review, 10, 213-220.

Fisher, R. A. (1930). The genetical theory of natural selection. Oxford, England: Clarendon.

Foley, R. (1995-1996). The adaptive legacy of human evolution: A search for the environment of evolutionary adaptedness. Evolutionary Anthropology, 4(6), 194-203.

Futuyma, D. J. (1998). Evolutionary biology (3rd ed.). Sunderland, MA: Sinauer.

Gould, S. J. (1977). Ontogeny and phylogeny. Cambridge, MA: Harvard University Press.

Hamilton, W. D. (1964). The genetical evolution of social behavior: Parts I and II. Journal of Theoretical Biology, 7, 1-16, 17-52.

Hamilton, W. D. (1996). Narrow roads of gene land: I. Evolution of social behaviour. Oxford, England: Freeman \& Spektrum and Oxford University Press.

Hartl, D. L., \& Clark, A. G. (1997). Principles of population genetics (3rd ed.). Sunderland, MA: Sinauer.

Hass, R. G., Chaudhary, N., Kleyman, E., Nussbaum, A., Pulizzi, A., \& Tison, J. (2000). The relationship between the theory of evolution and the social sciences, particularly psychology. In D. LeCroy \& P. Moller (Eds.), Annals of the New York Academy of Sciences: Vol. 907. Evolutionary perspectives on human reproductive behavior (pp. 1-20). New York: New York Academy of Sciences.

Ketelaar, T., \& Ellis, B. J. (2000a). Are evolutionary explanations unfalsifiable? Evolutionary psychology and the Lakatosian philosophy of science. Psychological Inquiry, 11, 1-21.

Ketelaar, T., \& Ellis, B. J. (2000b). On the natural selection of alternative models: Evaluation of explanations in evolutionary psychology. Psychological Inquiry, 11, 56-68.

Kimura, M. (1969). The rate of molecular evolution considered from the standpoint of population genetics. Proceedings of the $\mathrm{Na}$ tional Academy of Science USA, 63, 1181-1188.

Krebs, J. R., \& Davies, N. B. (1987). An introduction to behavioural ecology. Oxford, England: Blackwell Scientific.

Laland, K. N., Odling-Smee, F. J., \& Feldman, M. W. (1996). The evolutionary consequences of niche construction: A theoretical investigation using two-locus theory. Journal of Evolutionary Biology, 9, 293-316.

Laland, K. N., Odling-Smee, F. J., \& Feldman, M. W. (2000). Niche construction, biological evolution, and cultural change. Behavioral and Brain Sciences, 23, 131-175.

Lewontin, R. C. (1974). The genetic basis of evolutionary change. Chicago: University of Chicago Press.

Lewontin, R. C. (1983). Gene, organism, and environment. In D. S. Bendall (Ed.), Evolution from molecules to men (pp. 273-285). New York: Cambridge University Press.

Lewontin, R. C. (1996). Evolution as engineering. In J. Collado-Vides, B. Magasanik, \& T. F. Smith (Eds.), Integrative approaches to molecular biology (pp. 1-10). Cambridge, MA: MIT Press.

Lloyd, E. A. (1999). Evolutionary psychology: The burdens of proof. Biology and Philosophy, 14, 211-233.

Mayr, E. (1983). How to carry out the adaptationist program. American Naturalist, 121, 324-334.

Miller, G. (2000a). How to keep our metatheories adaptive: Beyond Cosmides, Tooby, and Lakatos. Psychological Inquiry, 11, $42-46$.

Miller, G. (2000b). Mental traits as fitness indicators: Expanding evolutionary psychology's adaptationism. In D. LeCroy \& P. Moller (Eds.), Annals of the New York Academy of Sciences: Vol. 907. Evolutionary perspectives on human reproductive behavior (pp. 62-74). New York: New York Academy of Sciences.

Pollack, E. (1978). With selection for fecundity the mean fitness does not necessarily increase. Genetics, 90, 383-389.

Richardson, R. C. (2000). Epicycles and explanations in evolutionary psychology. Psychological Inquiry, 11, 46-49.

Rozin, P. (2000). Evolution and adaptation in the understanding of behavior, culture, and mind. American Behavioral Scientist, 43, 970-986.

Shapiro, L., \& Epstein, W. (1998). Evolutionary theory meets cognitive psychology: A more selective perspective. Mind and Language, 13, 171-194.

Symons, D. (1995). Beauty is in the adaptations of the beholder: The evolutionary psychology of human female sexual attractiveness. In P. Abramson \& S. Pinkerton (Eds.), Sexual nature/sexual culture (pp. 80-118). Chicago: University of Chicago Press.

Tooby, J., \& Cosmides, L. (1992). The psychological foundations of culture. In J. H. Barkow, L. Cosmides, \& J. Tooby (Eds.), The adapted mind: Evolutionary psychology and the generation of culture (pp. 19-136). New York: Oxford University Press.

Tooby, J., \& Cosmides, L. (1998). Evolutionizing the cognitive sciences: A reply to Shapiro and Epstein. Mind and Language, 13, 195-204.

Tooby, J., \& DeVore, I. (1987). The reconstruction of hominid behavioral evolution through strategic modeling. In W. G. Kinzey (Ed.), The evolution of human behavior: Primate models (pp. 183-237). Albany: State University of New York Press.

Uyenoyama, M., \& Feldman, M. W. (1981). On relatedness and adaptive topography in kin selection. Theoretical Population Biology, 19, 87-123.

Williams, G. C. (1966). Adaptation and natural selection. Princeton, NJ: Princeton University Press.

Williams, G. C. (1992). Natural selection: Domains, levels, and challenges. New York: Oxford University Press.

Williams, G. C., \& Nesse, R. M. (1991). The dawn of Darwinian medicine. Quarterly Review of Biology, 66, 1-22.

Wright, S. (1931). Evolution in Mendelian populations. Genetics, 16, 97-159.

Young, J., \& Persell, R. (2000). On the evolution of misunderstandings about evolutionary psychology. In D. LeCroy \& P. Moller (Eds.), Annals of the New York Academy of Sciences: Vol. 907. Evolutionary perspectives on human reproductive behavior (pp. 218-223). New York: New York Academy of Sciences. 